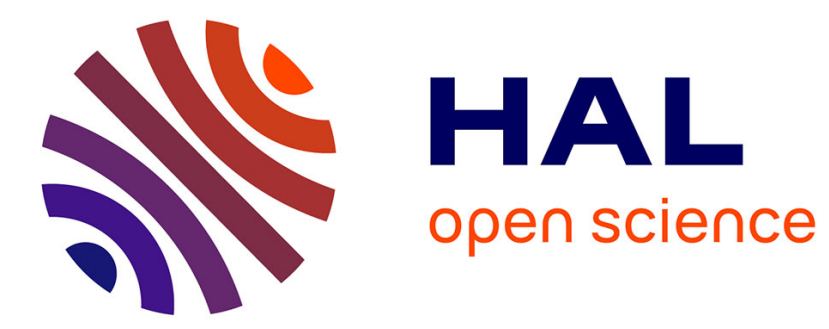

\title{
Dynamics of the shape transition of a magnetic ferrofluid drop
}

\author{
J.-C. Bacri, D. Salin
}

\section{To cite this version:}

J.-C. Bacri, D. Salin. Dynamics of the shape transition of a magnetic ferrofluid drop. Journal de Physique Lettres, 1983, 44 (11), pp.415-420. 10.1051/jphyslet:019830044011041500 . jpa-00232211

\section{HAL Id: jpa-00232211 https://hal.science/jpa-00232211}

Submitted on 1 Jan 1983

HAL is a multi-disciplinary open access archive for the deposit and dissemination of scientific research documents, whether they are published or not. The documents may come from teaching and research institutions in France or abroad, or from public or private research centers.
L'archive ouverte pluridisciplinaire HAL, est destinée au dépôt et à la diffusion de documents scientifiques de niveau recherche, publiés ou non, émanant des établissements d'enseignement et de recherche français ou étrangers, des laboratoires publics ou privés. 
Classification

Physics Abstracts

$75.50 \mathrm{M}-67.65-64.70$

\title{
Dynamics of the shape transition of a magnetic ferrofluid drop
}

\author{
J.-C. Bacri and D. Salin \\ Laboratoire d'Ultrasons (*), Université Pierre et Marie Curie, \\ Tour 13, 4, place Jussieu, 75230 Paris Cedex 05, France
}

(Reçu le 21 janvier 1983, accepté le 12 avril 1983)

\begin{abstract}
Résumé. - Au-delà d'un certain champ magnétique seuil, la forme ellipsoïdale allongée de gouttes magnétiques devient instable : la goutte passe d'une forme peu allongée à une forme beaucoup plus allongée. Nous avons fait la théorie de la dynamique critique de cette transition du premier ordre, c'est-à-dire que nous avons établi le comportement du temps d'établissement de l'instabilité au voisinage du seuil en champ magnétique. Nous avons mesuré la divergence critique du temps caractéristique de l'établissement de cette instabilité dans des gouttes magnétiques de ferrofluides. Les résultats expérimentaux sont en accord avec notre modèle théorique.
\end{abstract}

\begin{abstract}
The prolate ellipsoidal shape of a magnetic fluid drop becomes unstable at a certain magnetic field threshold : the drop jumps from a slightly elongated shape to a much more elongated shape. We describe theoretically the dynamics of this first order transition i.e. the relaxation time of this instability around the critical magnetic threshold. The critical divergence of the characteristic time of this instability, that we have measured in ferrofluid magnetic drops, is in reasonable agreement with our theoretical model.
\end{abstract}

\section{Introduction.}

It was recently shown, both theoretically and experimentally [1-4], that when a fluid containing an immiscible magnetic fluid drop is subjected to a magnetic field, the drop deforms into a prolate ellipsoid whose axis of revolution is along the field direction. This deformation may be simply understood as resulting from a balance between magnetic energy and interfacial tension energy $[4,5]$.

Moreover, depending on the relative values of the permeability of the two fluids, a shape instability can occur. Above a certain magnetic field threshold $H_{1}$ the prolate ellipsoidal shape of the drop becomes unstable, i.e. the drop jumps from a slightly elongated shape to a much more elongated shape [6]. On decreasing the magnetic field the reverse feature occurs for a smaller threshold $\mathrm{H}_{2}$. In a previous paper [6], we reported on the statics of this transition in ferrofluid agglomerate magnetic drops [7]. It was mentioned that the deformation of the drop in the vicinity of $\mathrm{H}_{1}$ or $\mathrm{H}_{2}$ can take a long time (up to a few minutes). In the present paper, we focus our attention on the critical dynamics of the transition near the threshold $H_{1}=H_{\mathrm{c}}$; i.e. the time dependence of the deformation as the magnetic field jumps from $H_{\mathrm{c}}$ to a field value $H$ just above $H_{\mathrm{c}}$.

(*) Associated with the Centre National de la Recherche Scientifique. 


\section{Theory.}

Let us first recall some aspects of the statics of the transition. The shape of a magnetic incompressible fluid drop of permeability $\mu_{2}$ in a fluid of permeability $\mu_{1}$ is due to a competition between its magnetic energy $E_{\mathrm{M}}$ and its interfacial energy $E_{\mathrm{s}}$. As discussed elsewhere [6], we assume a prolate ellipsoidal shape (semi major axis $a$, semi minor axis $b$ ) for the drop as it gives analytic expressions for $E_{\mathrm{s}}$ and $E_{\mathrm{M}}$. For a constant volume $V=\frac{4 \pi R_{0}^{3}}{3}\left(R_{0}\right.$ radius of the spherical drop in zero field) and a given magnetic field $H$, the shape of the drop (i.e. its elongation ratio $e=a / b$ ) is given by the minimization of the total energy $E_{\mathrm{T}}=E_{\mathrm{M}}+E_{\mathrm{s}}$ with respect to $e$. In fact this minimization gives the extrema of $E_{\mathrm{T}}$ but the stable shape of the drop corresponds to the minimum of lowest energy. This was discussed in our previous paper [6]. We give here a graph (Fig. 1) of $E_{\mathrm{T}}$ versus $e=a / b$ for $H$ in the vicinity of $H_{\mathrm{c}}\left(=H_{1}\right)$ when the instability of deformation can occur $\left(\mu_{2} / \mu_{1} \gtrsim 20, \sigma\right.$ interfacial tension) :

i) $h=\left(H-H_{\mathrm{c}}\right) / H_{\mathrm{c}}=-0.1, H$ is much smaller than $H_{\mathrm{c}}$ (but larger than $\left.H_{2}\right)$ the slightly elongated shape $(e \sim 1.9)$ is stable while the elongated shape is metastable.

ii) For a magnetic field $H$ just below $H_{\mathrm{c}}(h=-0.05)$, there are also two minima of $E_{\mathrm{T}}$. The slightly elongated shape (which was stable at low field) of the drop is metastable while the much more elongated one is stable.

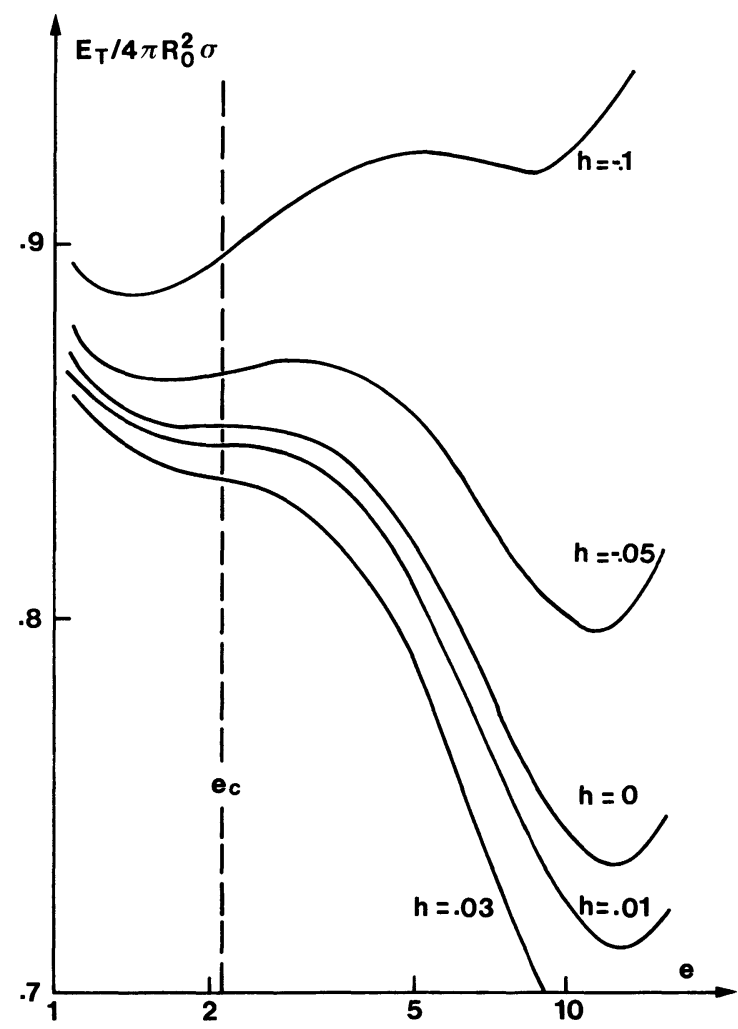

Fig. 1. - Semi-log plot of the normalized energy $E_{\mathrm{T}} / 4 \pi R_{0}^{2} \sigma$ versus the elongation $e$ for $\alpha=0.027$, $e_{\mathrm{c}}=2.10$. The different curves correspond to different values of the reduced field $\left(h=\left(H-H_{\mathrm{c}}\right) / H_{\mathrm{c}}\right)$. 
iii) For $H=H_{\mathrm{c}}$, the slightly elongated shape of the drop $(e \sim 2)$ becomes unstable, and it must go to the stable much more elongated shape.

iv) For $H>H_{\mathrm{c}}(h=0.01$ and $h=0.03)$, only one very elongated shape is stable.

The critical field $H_{\mathrm{c}}$ and the critical elongation $e_{\mathrm{c}}$ are defined as the values of $H$ and $e$, respectively, where the plot of $E_{\mathrm{T}}$ against $e$ shows an inflection. Note that for all the magnetic parameters of the problem the value of $e_{\mathrm{c}}$ is close to 2 . The jump from a slightly elongated shape ( $\left.e_{\mathrm{c}}\right)$ to a much more elongated shape $(e>10)$, for $H$ just above $H_{\mathrm{c}}$, is a first order transition.

The study of the dynamics of this transition is the study of the time dependence of the departure of $e$ from its critical value $e_{\mathrm{c}}$ for $H$ just above $H_{\mathrm{c}}$. The speed of this departure depends on the speed at which the curve $E_{\mathrm{T}}$ versus $e$ goes away from the inflection as $H$ increases from $H_{\mathrm{c}}$. The time dependence of $e$ is given by a Euler-Lagrange equation :

$$
\frac{\partial \dot{E}_{\eta}}{\partial \dot{e}}=\frac{\partial E_{\mathrm{T}}}{\partial e}
$$

where the inertial term has been neglected (we focus our attention on the overdamped deformation of the drop [8]). $\dot{E}_{\eta}$ is the rate of viscous dissipation and is only precisely known for a very nearly spherical ellipsoid [9]. Anyway from dimensionality arguments we can write it as [8] :

$$
\dot{E}_{\eta}=-\frac{1}{2} \eta R_{0}^{3} f(e) \dot{e}^{2}
$$

where $f(e)$ is an unknown function of the shape $e(f>0)$ but with a priori no drastic dependence on $e$ in the vicinity of $e_{\mathrm{c}}, \dot{e}=\partial e / \partial t$ and $\eta$ is an effective viscosity of the drop in the fluid [11]. If the surrounding fluid can be assumed inviscid compared to the fluid drop, $\eta$ is the viscosity of the fluid drop.

As we are interested in the behaviour of $e$ in the vicinity of $e_{\mathrm{c}}$ and for $H$ in the vicinity of $H_{\mathrm{c}}$, we have only to retain on the right hand side of equation (1) the first non trivial terms of the expansion of $E_{\mathrm{T}}(H, e)$ around $E_{\mathrm{T}}\left(H_{\mathrm{c}}, e_{\mathrm{c}}\right)$ taking into account the critical condition (inflection) :

$$
D \dot{e}=A h+B\left(e-e_{c}\right)^{2}
$$

with

$$
D=-\eta R_{0}^{3} f\left(e_{\mathrm{c}}\right), \quad A=\left.H_{\mathrm{c}} \frac{\partial^{2} E_{\mathrm{T}}}{\partial H \partial e}\right|_{\substack{H=H_{\mathrm{c}} \\ e=e_{\mathrm{c}}}}, \quad B=\left.\frac{1}{2} \frac{\partial^{3} E_{\mathrm{T}}}{\partial e^{3}}\right|_{\substack{H=H_{\mathrm{c}} \\ e=e_{\mathrm{c}}}} \cdot
$$

Integration of (3) with the condition $e-e_{\mathrm{c}}=0$ for $t=0$ gives :

$$
e-e_{\mathrm{c}}=\sqrt{\frac{A}{B} h} \operatorname{tg} \frac{t}{\tau} \text { with } \frac{1}{\tau}=\frac{\sqrt{A B}}{D} \sqrt{h}
$$

This means that $e=a / b$ increases from $e_{\mathrm{c}}$ according to a tangent function with a characteristic time $\tau$. This characteristic time $\tau$ diverges at the transition (i.e. goes to infinity) with a critical mean field, exponent $-0.5\left(\tau=\tau_{0} h^{-0.5}\right)$.

From the explicit form of $E_{\mathrm{T}}$ we find that $\tau_{0}$ is of the order of $\eta R_{0} / \sigma$ which is the hydrodynamic deformation time of the drop (without inertial effects). Note that it is not necessary to expand the right hand side of equation (3) to higher order because the failure of (3) comes essentially from the unknown viscous dissipation function. In order to determine (experimentally) the value of $D$ (i.e. $\eta f(e))$ we note that we can exactly calculate the value of the slope at the origin of $e-e_{\mathrm{c}}$ 
versus $t$ whatever $H\left(H>H_{\mathrm{c}}\right)$ may be : we have only to retain on the right hand side of equation(1) the first term of the expansion of $E_{\mathrm{T}}(H, E)$ around $E_{\mathrm{T}}\left(H, e_{\mathrm{c}}\right)$; taking into account the explicit form of $E_{\mathrm{T}}[6]$ we get :

$$
e-e_{\mathrm{c}}=S t ; \quad S=\frac{A}{2 D}\left(\frac{H^{2}}{H_{\mathrm{c}}^{2}}-1\right) .
$$

Equation (4) can be rewritten in the more convenient form :

$$
e-e_{\mathrm{c}}=S \tau \operatorname{tg} \frac{t}{\tau}
$$

which shows that the only critical variable is $\tau$. Our treatment is also applicable to the threshold $H_{2}$ where the elongated agglomerate reabsorbs its ends, but as was already mentioned [6] as the drop becomes more and more slender its shape becomes less and less ellipsoidal and we no longer have an analytic expression for its energy $E_{\mathrm{T}}$.

As a summary of the theoretical predictions : assuming a prolate ellipsoid shape of the drop allows a calculation of the parameters $A, B$ of the theory. The dissipation coefficient $D$ can be deduced from the measurement of the slope at the origin of $e-e_{\mathrm{c}}$ versus $t$. Therefore we have all the parameters which can give a quantitative test of the dynamics of this first order transition.

\section{Experiment.}

The magnetic fluid used is a ferrofluid obtained through a chemical process without surfactant [11]. Ferrofluids are colloidal suspensions of ferromagnetic grains of a typical size of $100 \AA$. The concentration in ionic iron is molar. The preparation of ferrofluid agglomerate drops has already been described [4] : the nearly spherical $(H=0)$ agglomerates consist of a more concentrated (in magnetic grains) phase in a less concentrated phase. The density difference between the two phases is a few per cent. Their radii are between 2 and $20 \mu \mathrm{m}$. The microscope observation is made with a black and white video Camera (horizontal definition : 500 points) with a television screen. The time is given by a $1 / 100 \mathrm{~s}$ video chronometer on each picture : due to the television process our time accuracy is $1 / 25 \mathrm{~s}$. Each experiment is recorded with a VHS magnetoscope and then analysed picture after picture. The ratio $e=a / b$ is deduced from a measurement of $a$ along the horizontal direction and the assumption of constant volume of the ellipsoid $\left(a b^{2}=R_{0}^{3}\right)$. The accuracy of the measured $e$ is better than $5 \%$. The sensitivity in field measurements has been improved : through current measurements we can measure a field variation as small as $2 \times 10^{-4}$ oersted. For each agglomerate we do a field cycle [6] (e versus $H^{2}$ ) in order to determine $H_{\mathrm{c}}, e_{\mathrm{c}}, \alpha$ and $\sigma$. In the experiment reported here, the agglomerate selected has a radius $R_{0}=10.5 \mu \mathrm{m}$, a field threshold $H_{1}=H_{\mathrm{c}}=1.39$ oersted with an elongation $e_{\mathrm{c}}=2.10 . \alpha=0.0270\left(\mu_{2} / \mu_{1} \simeq 38\right)$ and the interfacial tension is then $\sigma=6.1 \times 10^{-4} \mathrm{cgs}$. From these values of $H_{\mathrm{c}}$ and $e_{\mathrm{c}}$ we compute $A=$ $-2.660 \sigma R_{0}^{2}$ and $B=-0.320 \sigma R_{0}^{2}$.

Our experimental procedure is the following : for each value of $h$ the shape of the drop is stabilized with a field value just below $H_{\mathrm{c}}(e=2)$; we increase linearly the field up to the desired value of $H$ in less than $0.1 \mathrm{~s}$. The origin of time is taken to be when $e=e_{\mathrm{c}}$. Before analysing each experiment, we record all the experiments from large jumps in field values down to the transition $(h=0)$ which is determined to an accuracy of $2 \times 10^{-4}$. We have done 31 recordings corresponding to 31 values of $h$ from 0.6 to $2 \times 10^{-4}$. For each value of $h$ we have measured the value of $e$ on each picture (every $1 / 25 \mathrm{~s}$ ). Variations of $e$ versus $t$ are given in figure 2 for six different values of $h$. As evidence for our picture the relaxation time of the instability increases drastically as $h$ decreases. Finally figure 2 shows for all values of $h$ that the elongation $e$ increases and saturates to its final value without any oscillation. This supports the neglect of inertial effects in the equation of motion (Eq. (1)). 


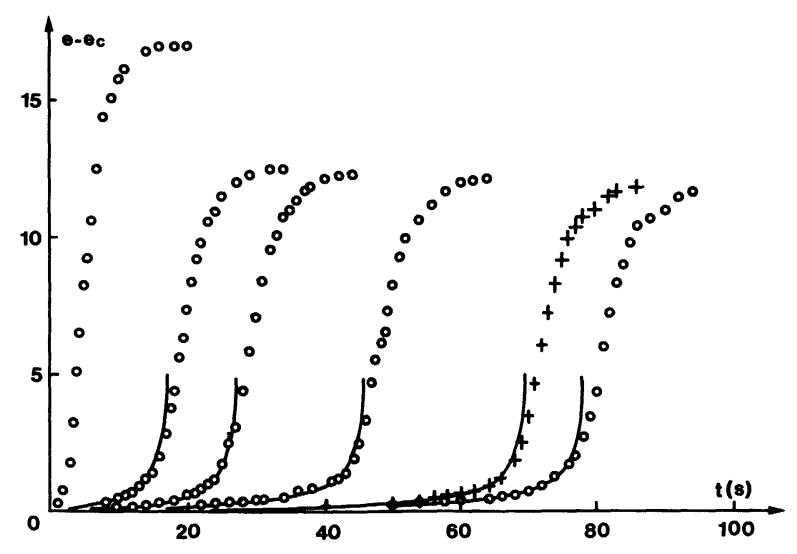

Fig. 2. - Experimental variations of the elongation versus the time $t$ for different values of $h$, from left to right, $h=1.4 \times 10^{-1}, h=1.6 \times 10^{-2}, h=6.8 \times 10^{-3}, h=3.2 \times 10^{-3}, h=1.9 \times 10^{-3}, h=8 \times 10^{-4}$. The curves, through the data are theoretical.

The first information comes from the slope $S$ at the origin, expression (5). Figure 3 is a $\log -\log$ plot of the value of $S$ versus $\frac{H^{2}}{H_{\mathrm{c}}^{2}}-1$. As expected we find a straight line of slope 1 ; we can then deduce the value of $A / 2 D=0.73 \mathrm{~s}^{-1} . A$ is known and we get $D \simeq-1.8 \sigma R_{0}^{2}$ and the effective viscosity times the unknown shape function $f\left(e_{\mathrm{c}}\right): \eta \cdot f\left(e_{\mathrm{c}}\right)=1.06$ Poise which is a reasonable order of magnitude for a concentrated ferrofluid [12]. $\left(f\left(e_{c}\right)\right.$ is then of the order of unity). As the slope at the origin is in accordance with our predictions, we next test the critical behaviour of $e-e_{\mathrm{c}}$. For each value of $h$, we find the value of $\tau$ which best fits our data to expression (6). Such fits are given in figure 2 where we can see that the $\operatorname{tg}(t / \tau)$ behaviour is observed quite far away from the critical elongation value $e_{\mathrm{c}}$ (up to $e-e_{\mathrm{c}} \sim 4-5$ ). This form is valid for $h \leqslant 3 \times 10^{-2}$. The values of $\tau$ versus $h$ are given in a $\log -\log$ plot in figure 4. The slope of the straight line through the date is -0.54 and one can vary it from -0.52 to -0.58 . This critical exponent, obtained over two decades in $h$ is in reasonable agreement with our theoretical prediction -0.5 . The last quantitative test of the theory is the value of the prefactor of the critical behaviour of $\tau$ (Eq. (4)). The

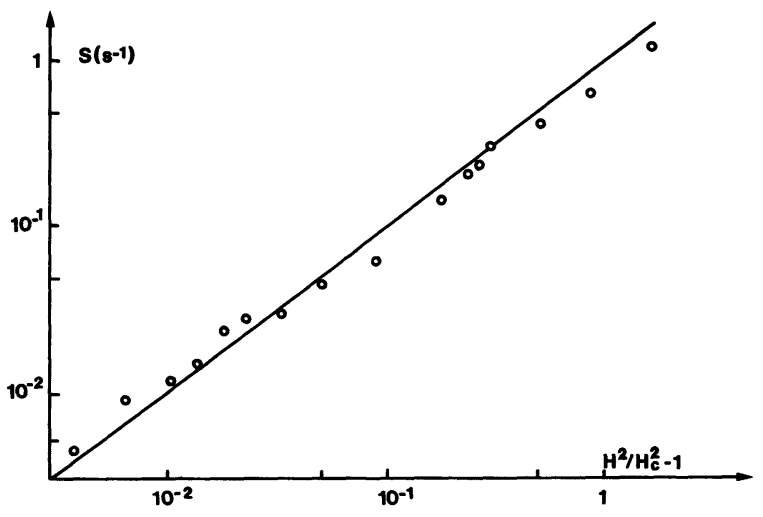

Fig. 3. - Log-log plot of the variation of the slope $S$ at origin (Fig. 2) versus $\frac{H^{2}}{H_{c}^{2}}-1$. The straight line has a slope 1 . 


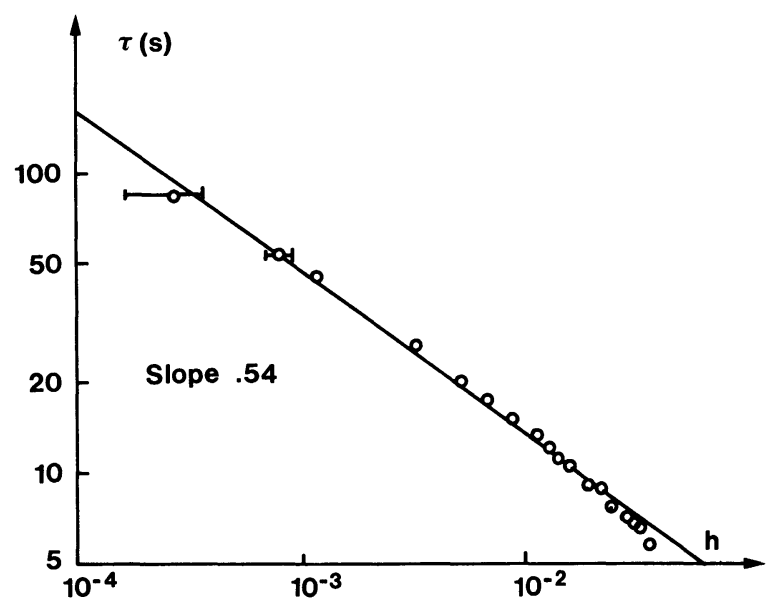

Fig. 4. - Log-log plot of the variation of the time $\tau$ of settlement of the instability versus $h$. The slope of the straight line through the data is 0.54 .

theoretical prediction, taking into account the value of $D$ obtained from the slope at the origin, is $\frac{1}{\tau}=\frac{\sqrt{A B}}{D} h^{0.5}=0.52 h^{0.5}$. Our experimental fit is $\frac{1}{\tau}=\frac{1}{\tau_{0}} h^{\alpha}$ with $\alpha=0.55 \pm 0.03$ and $\frac{1}{\tau_{0}}=$ $0.9 \pm 0.1$, in reasonable agreement with the theory.

\section{Conclusion.}

We have discussed the dynamics of the first order shape transition of a magnetic drop from a slightly elongated shape to a much more elongated one. We have measured the time dependence of the elongation $e$ for different field jumps $(h)$ close to the transition $\left(h\right.$ from 0.67 down to $2 \times 10^{-4}$ ). This time dependence is in reasonable agreement with our model for both the critical exponent of the characteristic time $\tau$ and its absolute values.

\section{Acknowledgments.}

We are greatly indebted to Prof. R. Massart for providing us with ferrofluid and to Prof. B. Caroli, Ch. Caroli and B. Roulet for stimulating discussions.

\section{References}

[1] Arkhipenko, V. I., Barkov, Yu. D. and Bashtovoi, V. G., Magnetohydrodynamics 14 (1978) 373.

[2] Arkhipenko, V. I., Barkov, Yu. D. and Bashtovoi, V. G., Magnetohydrodynamics 16 (1980) 228.

[3] Drozdova, Skrobotova and Chekanov, Magnetohydrodynamics 15 (1979) 12.

[4] Bacri, J.-C., Salin, D. and Massart, R., J. Physique-Lett. 43 (1982) L-179.

[5] Liao, W. H. and Krueger, D. A., J. Colloid. Interface Sci. 70 (1979) 564.

[6] BACRI, J.-C. and Salin, D., J. Physique-Lett. 43 (1982) L-649.

[7] General reference in Shliomis, M., Sov. Phys. Usp. 17 (1974) 153.

[8] Landau, L. D. and Lifshitz, E. M., Fluid Mechanics (Pergamon Press).

[9] Cox, R. G., J. Fluid Mechanics 37 (1969) 601.

[10] Taylor, G. I., Proc. R. Soc. A 146 (1934) 501.

[11] MASSART, R., IEEE Trans. Magn. 17 (1981) 1241.

[12] Rosensweig, R. E., Kaiser, R. and Miskolczy, G., Colloid Interface Sci. 29 (1969) 680. 